\title{
Clogging of Riverbeds - from Complex Field Conditions to Isolated Processes in the Laboratory
}

\author{
Markus NOACK \\ Faculty of Architecture and Civil Engineering, Karlsruhe University of Applied Science, \\ Karlsruhe, Germany \\ markus.noack@hs-karlsruhe.de
}

\begin{abstract}
The complex phenomenon riverbed clogging constitutes one reason for degraded riverine ecosystems. However, measuring or monitoring of clogging is challenging because of its multifaceted character. This keynote addresses clogging from different perspectives including field and laboratory aspects and shows recent developments in measuring techniques to gather relevant involved parameters and processes.
\end{abstract}

Keywords: riverbed clogging, colmation, field and laboratory methods, MultiPAC, gamma ray attenuation.

\section{INTRODUCTION}

Although clogging of riverbeds, also referred to colmation, has been studied for decades in the field as well as in hydraulic laboratories significant knowledge gaps exist to fully understand the complex and interacting physical and biogeochemical processes. The relevance of studying colmation and its single processes becomes obvious as it can have a tremendous impact on the hyporheic zone and the biotic community living there. In fact, riverbed clogging is suspected to be one reason for failing the target of the European Water Framework Directive in achieving the "good ecological status".

Riverbed clogging is also a matter of overlapping scales including macro-scale aspects on river basin level such as sediment delivery and hydrology but also micro-scale characteristics such as local topography, particle size/shape and the compaction of the riverbed. Moreover, clogging is a highly dynamic process.

These multifaceted issues are highly interesting but also challenging to cope with riverbed clogging, especially because of its high temporal and spatial variability but also because of the 
numerous multifaceted parameters and processes that need to be considered in measuring and monitoring riverbed clogging.

This keynote addresses the phenomena riverbed clogging from different perspectives and presents different methods to assess clogging in the field and in hydraulic laboratories.

\section{METHODS AND RESULTS}

For field applications, a so-called Multiparameter Approach for Colmation (MultiPAC) was developed to capture four key parameters in describing riverbed clogging on a local scale including particle size distribution, porosity, hydraulic conductivity and dissolved oxygen (Seitz 2020). The first results are very promising and prove that riverbed clogging cannot be described by single parameters. Especially the capability to gather vertical profiles allows the identification of clogged layers in the riverbed. However, to study the influence of single processes and parameters the complexity of riverbed clogging need to be simplified to be studied in hydraulic laboratories under controlled boundary conditions.

Therefore, a laboratory flume was designed to focus on sediment infiltration and accumulation only using a simplified bed made of spheres that can be arranged in different packings. Main objective of the experiments is to explore interactions between turbulences at the watersediment interface, interstitial flows and the progressive occlusion of pores with fine sediments that are fundamental to understand the dynamic behaviour of colmation processes. Therefore, the gamma ray attenuation method (GRA) was adapted and successfully tested to allow for nonintrusive measurements of sediment infiltration masses in vertical profiles (Mayar et al. 2020), which represents a precondition to study the dynamics of clogging processes. In future experiments, the measuring setup will be complemented by PIV measurements of surface flow and endoscopic PIV measurements of pore flow. These combined non-intrusive and simultaneously conducted measurements will lead to a unique dataset in describing the sediment infiltration and accumulation behaviour and allows for derivations of functional relationships between surface and subsurface processes to describe the interactions and dynamics of clogging processes on a fundamental and physical basis.

\section{CONCLUSIONS}

Despite the complicated phenomenon riverbed clogging, both the field and laboratory methods represents important advancements in studying the involved parameters and processes. While MultiPAC represents a step forward in measuring riverbed clogging in the field on a quantitative basis, the laboratory experiments with the GRA method enabled for the first time to study the dynamics of clogging given to its non-intrusive character. The combination with advanced PIV-techniques will further allow in-depth investigation, especially in quantifying interacting processes at the water-sediment interface.

Acknowledgments. The author presents his gratitude to Lydia Seitz and M. Assem Mayar for their work within their Ph.D. Thesis' and the Institute for Modelling Hydraulic and Environmental Systems at the University of Stuttgart, Germany where all the work and experiments have been realized. 
References

Mayar, M.A., G. Schmid, S. Wieprecht, and M. Noack (2020), Proof-of-concept for non-intrusive and undisturbed measurement of sediment infiltration masses using gamma-ray attenuation, J. Hydraul. Eng. 146, 5, DOI: 10.1061/(ASCE)HY.1943-7900.0001734.

Seitz, L. (2020), Development of new methods to apply a multiparameter approach - a first step towards the determination of colmation, Ph.D. Thesis No. 276, University of Stuttgart, Stuttgart, Germany, DOI: 10.18419/opus-11249.

Received 22 March 2021

Accepted 12 April 2021 